\title{
Investigation on the optimal magnetic field of a cusp electron gun for a W-band gyro-TWA
}

\author{
Liang Zhang, ${ }^{1, \text { a) }}$ Wenlong He, ${ }^{1}$ Craig R. Donaldson, ${ }^{1}$ and Adrian W. Cross ${ }^{1}$ \\ Department of Physics, SUPA, University of Strathclyde, Glasgow, G4 ONG, Scotland, \\ $U K$
}

(Dated: 24 April 2018)

High efficiency and broadband operation of a gyrotron traveling wave amplifier (gyro-TWA) requires a highquality electron beam with low-velocity spreads. The beam velocity spreads are mainly due to the differences of the electric and magnetic fields that the electrons withstand the electron gun. This paper investigates the possibility to decouple the design of electron gun geometry and the magnet system while still achieving optimal results, through a case study of designing a cusp electron gun for a W-band gyro-TWA. A global multiple-objective optimization routing was used to optimize the electron gun geometry for different predefined magnetic field profiles individually. Their results were compared and the properties of the required magnetic field profile are summarized.

Keywords: gyrotron traveling wave amplifier, cusp electron gun, large orbit electron beam, cusp magnetic field

\section{INTRODUCTION}

Gyro-devices are high power microwave sources based on the mechanism of cyclotron resonance maser instability ${ }^{1}$, in which the electrons gyrate in a constant magnetic field. In gyro-devices, the electrons are able to interact with the fast waves, therefore they attract significant interest due to the power demands in the millimeter and sub-millimeter wavelength ranges for applications such as communications, RADAR, dynamic nuclear polarization, and so on.

Two typical categories of electron beams are used for the beam-wave interaction in gyro-devices. One is that each electron gyrates at cyclotron frequency around its own different guiding center and drift gradually while bunching in the transverse phase space in the presence of a strong axial magnetic field. This can be realized by using a magnetron injection gun ${ }^{2}$. The other one is that the electrons gyrate with an axis-encircling orbit, which is called a large orbit beam $(\mathrm{LOB})^{3}$. When interacting with electromagnetic wave the LOB has mode selective capability which mitigates the problem of mode competition. Such a beam only interacts with the $\mathrm{TE}_{m n}$ modes with the azimuthal index $m$ equal to the harmonic number, $s$, of the electron cyclotron mode. The gyro-devices operating at a harmonic cyclotron frequency have the advantage of reducing the required magnetic field strength, which becomes increasing important at millimeter and sub-millimeter wavelengths.

The generation of the LOB can be realized by adding a kicker coil, which introduces a transverse force to the electron beam generated by a Pierce type gun ${ }^{4,5}$. However in practice, it is difficult to realize such nonsymmetrical magnetic field and the electron beam quality

\footnotetext{
a)Electronic mail: liang.zhang@strath.ac.uk
}

is hard to control. A better method is to keep the magnetic field symmetrical with a reversal in the direction of the field in the path of the electron beam. From the conservation of the canonical angular momentum, the electrons will encircle around the longitudinal axis at the exit of the field reversal ${ }^{6}$. The electron guns using an adiabatic field reversal were firstly employed in ${ }^{7-9}$. Their drawbacks include: small cathode radius, difficult to achieve short field transition length and particular field configurations. It is more attractive to use a smooth cusp magnetic field ${ }^{10-13}$. This configuration does not require magnet poles therefore simplifying the system. The magnetic field reversal point can be close to the cathode where the electron beam has not been fully accelerated. The beam energy is low, therefore a small reversed magnetic field strength is needed. The drawbacks of the smooth cusp configuration is that the compression region for the electron beam is relatively long and adds extra length to the whole system and alignment can be more difficult.

The trajectories of the electrons can be numerically calculated if the electric and magnetic fields are known. However, it is still a challenge to design an ideal cus$\mathrm{p}$ electron gun. So far none synthesis method has been proposed to derive the optimal cathode and anode geometries, as well as the optimal magnetic field configuration. The design of a new cusp electron gun usually results in altering an existing one, or parameters scanning to study the effects from single or a limited number of free parameters. This not only requires a lot experience but also is time consuming as there are many free parameters that need to be taken into account. Moreover, the designed cusp gun may still not be the global optimum.

Both the electric and magnetic fields affect the performance of the designed cusp gun. The electric field inside the electron gun is determined by the geometries of the cathode and anode. The magnetic field is governed by the coil setup. In most of cases, their effects are coupled which make the design a challenging task.

The design process can be greatly simplified if the de- 
sign of the magnetic field and the electron gun geometry can be decoupled. Experience from previous studies ${ }^{10-12}$ showed that to achieve an optimal performance, an optimal magnetic field can be found for a known electric field configuration, and vice versa. However it was found that it was easier to achieve an optimal result by optimizing the electron gun geometry instead of the magnetic field system.

An interesting question arises. Can a global optimum always be reached through optimizing the electron gun geometry with a given magnetic field, and what properties does the magnetic field need to have? Unfortunately the question is unlikely answered from theory because the dynamics of the electrons in the cusp gun is complicated. Normally no analytical solutions exist for the electric field.

In this paper, this question was studied numerically based on a case design of a cusp electron gun for a Wband gyrotron traveling wave amplifier (gyro-TWA). Different magnetic field profiles from real solenoid system$\mathrm{s}$ were designed, and a global optimization routing was used to find the optimal electron gun geometry for each magnetic field. The performances of the optimized cusp electron guns were compared. Several useful conclusions that could be universally applied to other cusp electron guns were drawn on the properties of the optimal magnetic field.

\section{THEORETICAL ANALYSIS AND NUMERICAL SIMULATIONS}

The motion of the electrons in axisymmetric electric and magnetic field follows the conservation of the canonical angular momentum, which can be expressed as ${ }^{14}$

$$
m_{0} \gamma r_{0}^{2} \dot{\theta}-\left(e r_{0}^{2} B_{0}\right) / 2=-\left(e r_{c}^{2} B_{c}\right) / 2
$$

where $e$ and $m_{0}$ are the electric charge and rest mass of an electron. $\gamma$ is the relativistic factor. $B_{c}, B_{0}$ are the magnetic fields at the cathode and the beam-wave interaction region respectively. $r_{c}, r_{0}$ are the electron beam radii at the cathode and the beam-wave interaction region. For an axis-encircling electron beam, it has

$$
\dot{\theta}=\omega_{0}=e B_{0} / m_{0} \gamma=v_{\perp} / r_{0}
$$

Therefore Eq. 1 can be rewritten as

$$
-r_{0}^{2} B_{0}=r_{c}^{2} B_{c}
$$

The alpha factor a, defined by $v_{\perp} / v_{z}$, can be written as

$$
a=\sqrt{-r_{c}^{2} \omega_{0} \omega_{c} /\left(V^{2}+r_{c}^{2} \omega_{0} \omega_{c}\right)}
$$

The relation between velocity spread and thickness of the emitter has been derived as Eq. 5 in Wang's paper ${ }^{8}$. Using the symbols defined in the paper, it can be rewritten as

$$
\triangle v_{\perp} / v_{\perp}=\left(2 r_{c}^{\prime} \triangle R\right) /\left(r^{2} B(z)-r_{c}^{\prime 2}\right)
$$

where $\Delta R$ is the thickness of the emitter. By applying $r^{2} B(z)=r_{0}^{2}\left(B_{0} / B_{c}\right)$ and $-r_{0}^{2} B_{0}=r_{c}^{\prime 2} B_{c}$ to Eq. 5, we will have

$$
\triangle v_{\perp} / v_{\perp}=\Delta R / r_{c}^{\prime}
$$

The electrons have the same energy at the end of the electron gun. Therefore $v_{\perp}^{2}+v_{z}^{2}=\left(1-\gamma^{-2}\right) c^{2}$ is a constant value. The velocity spread of the electron beam becomes

$$
\triangle v_{z} / v_{z}=\Delta v_{\perp} / v_{\perp} a^{2}=a^{2} \Delta R / r_{c}^{\prime}
$$

Eq. 6 and 7 are not precisely accurate as it is derived under two conditions: 1 ) the emitter surface is perpendicular to $Z$ axis, and 2) the off-axis magnetic field strength is calculated based on the first order approximation, i.e. $B_{z}(r, z)=B_{z}(r=0, z), B_{r}(r, z)=-0.5 r \cdot d B_{z}(r=$ $0, z) / d z$. However, they are useful guidance in choosing the radius of the emitter. On the other hand, the beam current for thermionic emission is given by

$$
I=\pi J r_{c} \Delta R
$$

In gyro-devices, the optimal values of beam voltage $V$, $B_{0}$ and $r_{0}$ are determined by the interaction circuit. The value of $r_{c}$ can be chosen as a balance of velocity spread and the $B_{c}$ value. A small $r_{c}$ results in a big inherit alpha spread, however a big $r_{c}$ will lead to a small $B_{c}$ which then requires precise control of the solenoid driving current.

The theoretical analysis offers a good starting point in electron gun design, however it does not provide useful information on optimal cathode geometry. Eq. 3 defines the required magnetic fields at the emitter position and the interaction position, however the optimal magnetic field profile between the two positions are still unknown.

In this study, the performance of the cusp electron gun was simulated numerically. MAGIC ${ }^{15}$ was chosen as the simulation tool. MAGIC is a particle-in-cell code which is different from the particle tracking solvers used in EGUN ${ }^{16}$ and CST Particle Studio ${ }^{17}$. It simulates a large number of particles at the same time and the spacecharge effect can therefore be more accurately simulated. Another advantage is that it offers a powerful command language interpreter and allows parameterization of the simulation. It can be invoked by other programs to achieve automatic optimization ${ }^{18}$. As the electric and magnetic fields are axisymmetric, a $2 \mathrm{D}$ simulation is a good representation of the physical situation. MAGIC $2 \mathrm{D}$ uses conformal mesh grids. It requires dense mesh grids to represent the electron gun geometry and get an accurate result. A large number of simulations were carried out and crosschecked with the existing design ${ }^{12}$ to verify the physical model. The influence of velocity and angular distributions of the emitted electrons from the thermionic cathode was also considered. A Maxwellian 
velocity distribution and sin-of-polar angle for the angular distribution was used in the simulation.

A general electron gun geometry was defined as the electric field simulation region, as shown in Fig. 1. It was derived from Vaughans synthesis of the Pierce gun with a hollow electron beam ${ }^{19}$. The whole structure was determined by 8 key parameters that are also labeled in Fig. 1. The emitter had a conical section. It was claimed that a curved shape would help to reduce the alpha spread ${ }^{20}$. However, for millimeter-wave gyro-devices operating with a lower order mode, the emitter thickness was relatively small (usually around $0.5 \mathrm{~mm}$ ) and the machining difficulty and tolerance would counteract the benefits of using a curved shape. The prominence of the bottom focusing electrode was to provide an additional mean to shape the electric field and hence the electron trajectories.

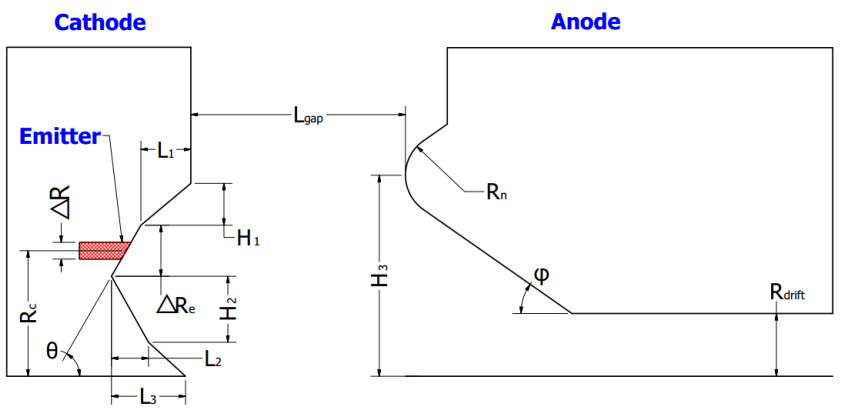

FIG. 1. (Color online) A general structure of a cusp electron gun.

A magnet system composed by four solenoids were used to generate the magnetic field. It included a main coil, two shim coils to compensate the field decrease at both ends of the main coil, and one reverse coil close to the electron gun to generate a magnetic field reversal, as shown in Fig. 2. The magnet system contained a number of free parameters, such as the coil radii, turns, layers, as well as their positions. In addition to achieving the required $B_{c}$ and $B_{0}$, its design also had to satisfy the capability of being able to be driven by a current that was readily available from a commercially available power supply. The magnet system was initially designed and optimized by analytical equations. Then the solenoid configurations were modeled and verified by the Pandira magnetic field solver ${ }^{21}$. The field in the electron gun region was imported into the MAGIC 2D simulations.

A typical magnetic field profile generated by the proposed solenoid system is shown in Fig. 3. The magnetic field could be divided into three regions, the field reversal region, the compression region, and the flat field region. The reversal region is most important because it has both electric and magnetic fields which strongly affect the velocity spread of the cusp electron gun. It influences the overall length of the compression region as well. In Fig. 3, a slope, $s=B_{c} / L$, was defined to quantitatively measure how quickly the magnetic field is changing at the

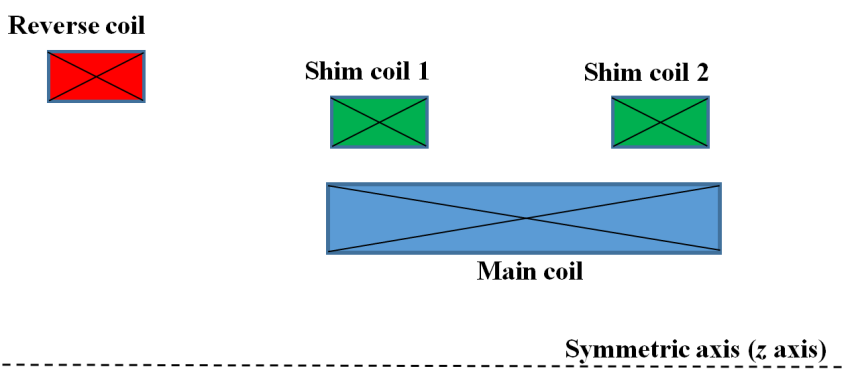

FIG. 2. (Color online) Solenoid setup to generate the magnetic field profile.

cusp region. Another parameter describing the deviation from the slope, the standard deviation of $B_{z}(z)-s z$ was used to measure the linearity of the magnetic field profile at the field reversal region, where $B_{z}(z=0)=0$ denotes the magnetic field cusp point.

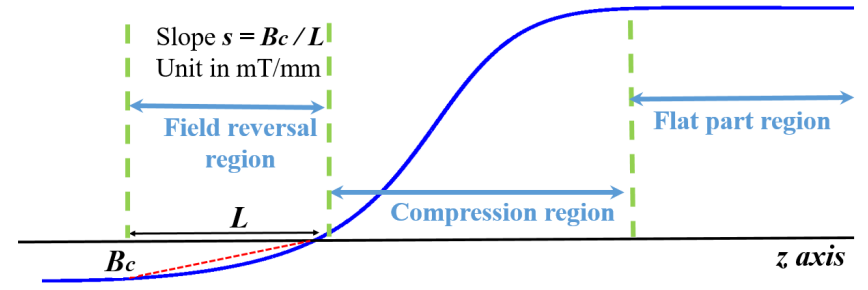

FIG. 3. (Color online) Definition of the magnetic field slope.

\section{OPTIMIZATION WITH DIFFERENT MAGNETIC PROFILES}

For gyro-devices operating at different frequencies, waveguide modes and cyclotron harmonic numbers, the required $V, B_{0}$ and $r_{0}$ are different. Therefore a case study has to be used and those parameters were assigned with specific values for numerical simulations. In this paper, these parameter are from a W-band gyro-TWA ${ }^{22}$. It operated at second harmonic of the cyclotron frequency and the operating frequency range was 90-100 GHz. The values of desired beam parameters are listed in Table 1 .

\begin{tabular}{ll}
\hline Beam voltage $V$ & $40 \mathrm{kV}$ \\
\hline Beam current $I$ & $1.5 \mathrm{~A}$ \\
\hline Beam alpha $a$ & 1.1 \\
\hline Harmonic number $s$ & 2 \\
\hline$B_{0}$ & $1.82 \mathrm{~T}$ \\
\hline$R_{c}$ & $6.0 \mathrm{~mm}$ \\
\hline$\Delta R$ & $0.5 \mathrm{~mm}$ \\
\hline$B_{c}($ can be adjusted in optimizations $)$ & $3.6 \mathrm{mT}$ \\
\hline
\end{tabular}

TABLE I. List of beam parameters

As the field reversal region played the most important role. To cover as much possible magnetic field profiles 
and to achieve a universal conclusion from the numerical optimization a combination of different slopes and linearity at the cusp region were chosen, as shown in Fig. 4(a). The profiles had the same $B_{c}$ and $B_{0}$ values. Profile 1 and 2 have good linearity but with different slopes in the cusp region. Profiles 3 to 6 have a big difference in slope. Profiles 1 and 4, 2 and 5 have similar slopes, but they have different linearity at the cusp region. Fig. 4(b) shows the full magnetic field shapes. It clearly shows that both a lower slope and curve that is more linear will result in a longer compression region.

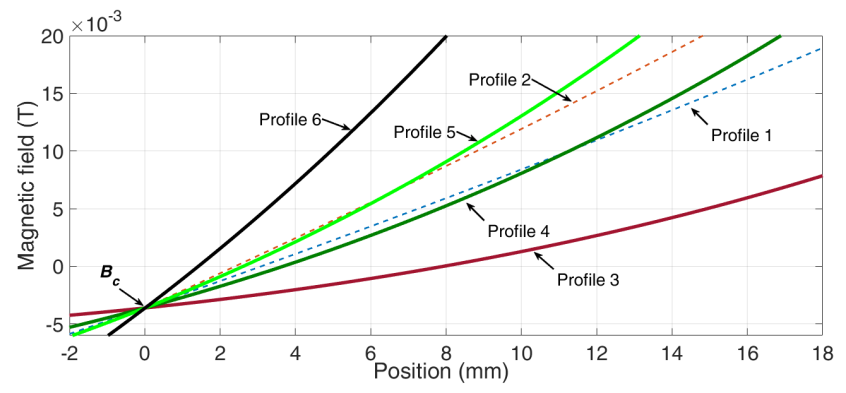

(a)

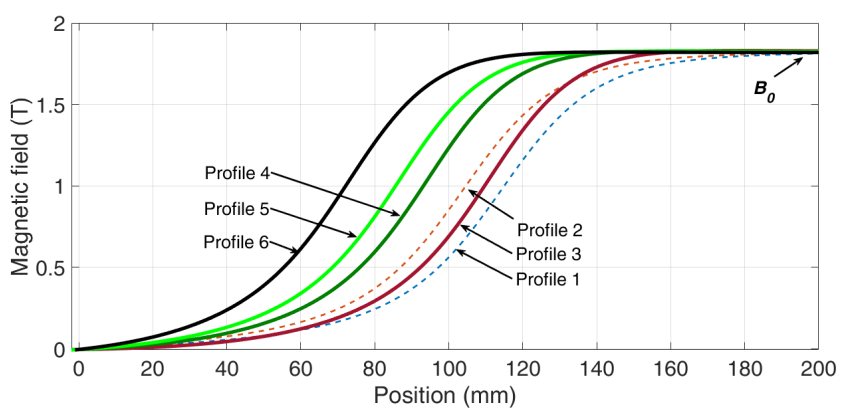

(b)

FIG. 4. Color online) Different magnetic field profiles in the cusp region (a), and from cathode to interaction region (b).

In the optimization, besides the 8 free variables in the gun geometry, another variable was used to shift the magnetic field within a small position range. This allowed a fine tuning of the alpha center to the desired value. A multiple-objective optimization was used to search for the global optimal geometry for individual magnetic field profiles. When the simulation of individual parameter set finished, the information of the electron cloud, including the positions and momentums, at the exit of the electron gun was exported for post processing. The velocities and alpha values were calculated and the distributions were plotted for evaluating the performance of the cusp electron gun.

The optimization routing had two evaluation functions. One was the combined value of the center velocity and alpha values with proper weights. The other one was their spreads which were defined by the ratio of the ful1 width at half maximum (FWHM) of the distribution curves to their central values. The optimal electron gun geometry would have the minimum alpha spread with its center close to the desired value. The other parameters, such as the emitted beam current, the beam transportation rate, and the average Larmor radius were also important. They were assigned different weights and treated as penalty factors to the final values of the evaluation functions.

\section{OPTIMIZATION RESULTS AND DISCUSSION}

The optimizations were run independently for individual magnetic field profiles. Each optimization contained 5600 iterations and took about two weeks on a 16-core computer. The optimized structure for each case was examined and the simulation results were analyzed. As an example, Fig. 5(a) and (b) shows the optimized geometry, the beam trajectory, the beam alpha value and its distribution for magnetic profile 1. A comparison of the alpha distributions for all the magnetic field profiles is shown in Fig. 6.

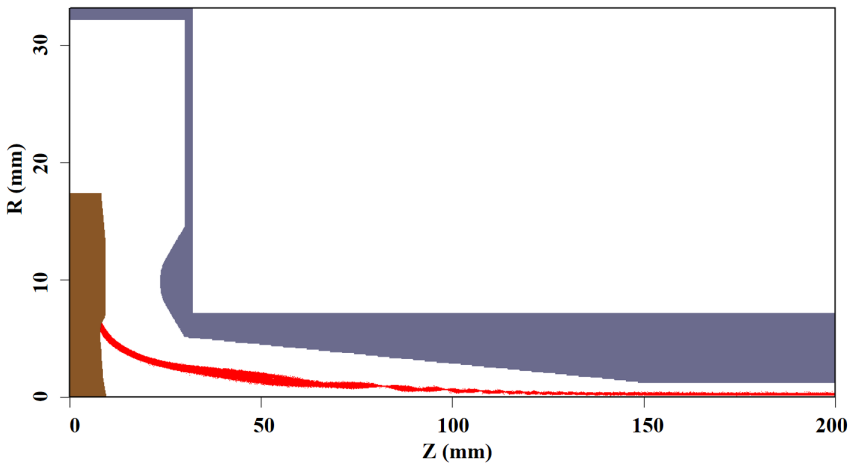

(a)

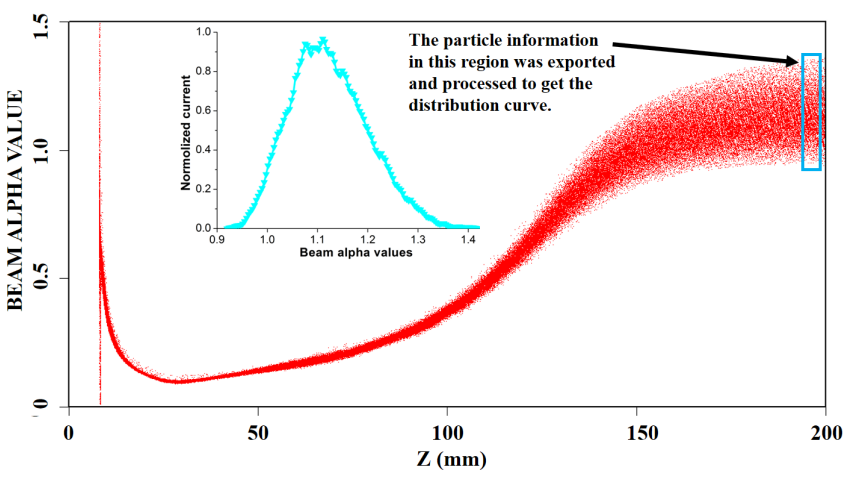

(b)

FIG. 5. Color online) The optimized geometry, the beam trajectory (a), the beam alpha value and its distribution (b) for magnetic field profile 1.

Magnetic field properties, including the slopes and the lengths of the compression regions, as well as the alpha spread of the optimized electron guns were summarized in Table 2. Several useful results were concluded as followed. 


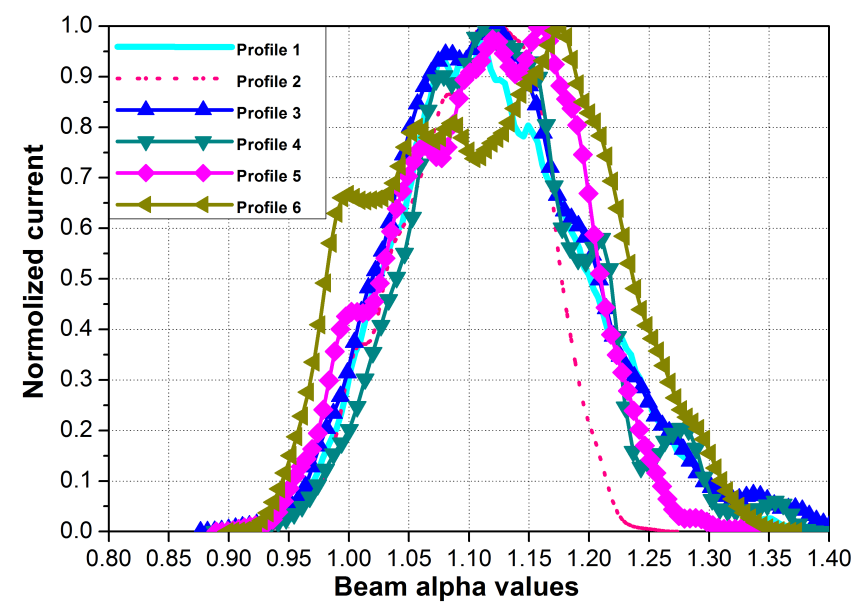

FIG. 6. (Colour online) The beam alpha distributions for different magnetic field profiles.

\begin{tabular}{|c|c|c|c|}
\hline $\begin{array}{l}\text { Magnetic } \\
\text { profile }\end{array}$ & $\begin{array}{l}\text { Slope } \\
(\mathrm{mT} / \mathrm{mm})\end{array}$ & $\begin{array}{l}\text { Length of } \\
\text { compres- } \\
\text { sion region } \\
(\mathrm{mm})\end{array}$ & $\begin{array}{l}\text { Alpha } \\
\text { spread }\end{array}$ \\
\hline 1 & 1.17 & 192.4 & $15.2 \%$ \\
\hline 2 & 1.51 & 183.7 & $14.4 \%$ \\
\hline 3 & 0.45 & 155.5 & $16.2 \%$ \\
\hline 4 & 1.00 & 144.5 & $15.9 \%$ \\
\hline 5 & 1.39 & 140.1 & $16.1 \%$ \\
\hline 6 & 2.56 & 131.3 & $23.4 \%$ \\
\hline
\end{tabular}

TABLE II. Summary of the optimization parameters

1) Magnetic field profiles 1 and 2 have smaller alpha spreads than the others, which means a good linearity of the magnetic field in the cusp region will help to improve the beam quality. This may be explained by the offaxis magnetic field at the emitter surface. If $B_{z}^{\prime}(0)$ is a constant value, the off-axis magnetic field components only have the first-order derivation. They are $B_{z}(r)=$ $B_{z}(0)$ and $B_{r}(r)=-r B_{z}^{\prime}(0) / 2$. The alpha spread of the electron beam is only caused by the $B_{r}(r)$ due to the thickness of the emitter $(\Delta R)$. The effect caused by higher order derivation does not exist and it is easier to find the optimal geometry.

2) The magnetic field was generated by a solenoid system as shown in Fig. 2. To generate a linearly profiled magnetic field at the cathode region, the radius of the reverse coil had to be large with a long distance between the reverse coil and the main coil. The magnet system would become bulky. As an example shown in Table 1, the compression region of profile 1 and 2 are $20 \%$ longer than the others.

3) Profiles 3 to 5 had very similar beam alpha distributions and only slightly bigger than profiles 1 and 2 . That showed in a reasonably large range of slope values, there existed an optimal electron gun geometry to achieve small alpha spread. However, if the slope is much larger, for example in profile 6 , the alpha spread increases signif- icantly. That could be due to the electron energy being low and relatively diverse when the cusp region is close to the emitter. If the magnetic field slope is larger, the resultant larger $B_{r}(r)$ value will have a bigger effect when the large orbit electron beam is forming.

4) Various magnetic field profiles can achieve similar optimal performance. The field slope at the field reversal region can be used to guide the design. As from the case study, a slope between 1.0-1.8 is a balanced choice for the W-band gyro-devices. A steep slope, larger than 2 , will have larger alpha spread which should be avoided. A lower slope than 1.0 should also work, however it will significantly increase the length of the compression region. For the cusp gun designed for gyro-devices operating at a different frequency, the slope range can be inversely proportional to $B_{0}$. At lower $B_{0}$, the value of $B_{c}$ will be slightly larger, the suggested slope value still works however it can be a slightly larger value. For the cusp gun operating at higher $B_{0}$, the beam compression ratio is relatively large, and a smaller slope will be more important to achieve a smaller alpha spread.

5) The optimization results show that in a cusp gun, the design of the electric and magnetic fields can be decoupled. The magnetic field can be designed first, and then to optimize the electron gun geometry based on the magnetic field. This can be a universal conclusion and suits for the cusp electron gun designed for gyrodevices operating at different frequencies and magnetic field strengths.

\section{v. CONCLUSION}

In this paper, through a case study of optimizing a cusp electron gun for a W-band gyro-TWA, it shows that different magnetic field profiles are able to achieve a similar alpha spread. Therefore the design of electron gun geometry and the magnet system can be decoupled. The magnetic field can be designed first. In the second step, the electron gun geometry can be optimized based on the designed magnetic field profile. An automatic optimization routing is recommended as the electron gun geometry contains many free parameters and the manual study would be labor intensive and require a lot of experience.

It was found that a relatively wide range of magnetic field profiles can be used. A good linearity at the field reversal region is preferred, if the larger size of the magnet system and extra alignment difficulty can be tolerated. General magnetic field profiles are also able to achieve similar performance with good linearity, as long as the slope is not too high. From experience, a magnetic field slope in the range of $1.0 \mathrm{C} 1.8$ is a balanced choice. A lower slope helps to reduce the assembly tolerance between the electron gun and the magnet system. However it increases the length of the compression region at the same time. 


\section{ACKNOWLEDGMENTS}

The authors would like to thank Engineering and Physical Sciences Research Council (EPSRC) U.K. (research Grant No. EP/K029746/1) and Science and Technology Facilities Council (STFC) (research Grant No. ST/P001890/1) for supporting this work.

${ }^{1}$ K. R. Chu, Rev. Mod. Phys. 76, 489 (2004).

${ }^{2}$ E. C. Morse, Laser and Particle Beams 15, 347C351 (1997).

${ }^{3}$ V. L. Bratman, Y. K. Kalynov, and A. É. Fedotov, Technical Physics 43, 1219 (1998).

${ }^{4}$ S. J. Cooke, A. W. Cross, W. He, and A. D. R. Phelps, Phys. Rev. Lett. 77, 4836 (1996).

${ }^{5}$ V. L. Bratman, A. W. Cross, G. G. Denisov, W. He, A. D. R. Phelps, K. Ronald, S. V. Samsonov, C. G. Whyte, and A. R. Young, Phys. Rev. Lett. 84, 2746 (2000).

${ }^{6}$ G. Schmidt, The Physics of Fluids 5, 994 (1962), http://aip.scitation.org/doi/pdf/10.1063/1.1706715.

${ }^{7}$ W. Lawson, Applied Physics Letters 50, 1477 (1987), https://doi.org/10.1063/1.97805.

${ }^{8} \mathrm{~J}$. Wang, Y. Luo, and N. C. Luhmann, IEEE Transactions on Plasma Science 38, 3356 (2010).

${ }^{9}$ S. G. Jeon, C. W. Baik, D. H. Kim, G. S. Park, N. Sato, and K. Yokoo, Applied Physics Letters 80, 3703 (2002), https://doi.org/10.1063/1.1480468.

${ }^{10}$ W. He, C. G. Whyte, E. G. Rafferty, A. W. Cross, A. D. R. Phelps, K. Ronald, A. R. Young, C. W. Robertson, D. C. Speirs, and D. H. Rowlands, Applied Physics Letters 93, 121501 (2008), https://doi.org/10.1063/1.2988259.

${ }^{11}$ C. R. Donaldson, W. He, A. W. Cross, A. D. R.
Phelps, F. Li, K. Ronald, C. W. Robertson, C. G. Whyte, A. R. Young, and L. Zhang, IEEE Transactions on Plasma Science 37, 2153 (2009).

${ }^{12}$ C. R. Donaldson, W. He, A. W. Cross, F. Li, A. D. R. Phelps, L. Zhang, K. Ronald, C. W. Robertson, C. G. Whyte, and A. R. Young, Appl. Phys. Lett. 96, 1412010501 (2010), https://doi.org/10.1063/1.3374888.

${ }^{13}$ C. H. Du, T. H. Chang, P. K. Liu, C. P. Yuan, S. J. Yu, G. F. Liu, V. L. Bratman, M. Y. Glyavin, and Y. K. Kalynov, IEEE Transactions on Electron Devices 59, 3635 (2012).

${ }^{14} \mathrm{M}$. J. Rhee and W. W. Destler, The Physics of Fluids 17, 1574 (1974).

${ }^{15}$ B. Goplen, L. Ludeking, D. Smith, and G. Warren, Computer Physics Communications 87, 54 (1995), particle Simulation Methods.

${ }^{16} \mathrm{~W}$. B. Herrmannsfeldt, Pulsed RF sources for linear colliders. Proceedings: Conference, Montauk, USA, Oct 2-7, 1994, AIP Conf. Proc. 337, 383 (1995).

${ }^{17}$ H. Spachmann and U. Becker, Nuclear Instruments and Methods in Physics Research Section A: Accelerators, Spectrometers, Detectors and Associated Equipment 558, 50 (2006), proceedings of the 8th International Computational Accelerator Physics Conference.

${ }^{18}$ L. Zhang, W. He, A. W. Cross, A. D. R. Phelps, K. Ronald, and C. G. Whyte, IEEE Transactions on Plasma Science 37, 390 (2009).

${ }^{19}$ J. R. M. Vaughan, IEEE Transactions on Electron Devices 34, 468 (1987)

${ }^{20}$ C. H. Du, X. B. Qi, B. L. Hao, T. H. Chang, and P. K. Liu, IEEE Electron Device Letters 36, 960 (2015).

${ }^{21} \mathrm{~J} . \quad \mathrm{H}$. Billen and L. M. Young, in Proceedings of International Conference on Particle Accelerators (1993) pp. $790-792$ vol.2.

${ }^{22}$ W. He, C. R. Donaldson, L. Zhang, K. Ronald, A. D. R. Phelps, and A. W. Cross, Phys. Rev. Lett. 119, 184801 (2017). 